\title{
Color Image Reduction by Minimizing Penalty Functions
}

\author{
Daniel Paternain, Aranzazu Jurio \\ Javier Fernandez and Humberto Bustince \\ Departamento de Automatica y Computacion \\ Universidad Publica de Navarra \\ Pamplona 31006, Spain \\ Email: daniel.paternain@unavarra.es \\ Web: http://giara.unavarra.es
}

\author{
Gleb Beliakov \\ School of Information Technology \\ Deakin University \\ Burwood 3125, Australia \\ Email: gleb@deakin.edu.au
}

\begin{abstract}
In image processing, particularly in image reduction, averaging aggregation functions play an important role. In this work we study the aggregation of color values (RGB) and we present an image reduction algorithm for RGB color images. For this purpose, we define and study aggregation functions and penalty functions in product lattices. We show how the arithmetic mean and the median can be obtained by minimizing specific penalty functions. Moreover, we study other penalty functions and we show that, in general, aggregation functions on product lattices do not coincide with the cartesian product of the corresponding aggregation functions. Finally, we make an experimental study where we test our reduction algorithm and we analyze the stability of the penalty functions in images affected by noise.
\end{abstract}

Index Terms-Image reduction, Product lattice, Penalty function.

\section{INTRODUCTION}

In image processing, image reduction consists in reducing the dimension of an image while keeping as much information as possible from it. Image reduction can be used to accelerate computations on an image (i.e. transformations), or just to reduce the cost of its storage or transmission.

For reducing grayscale images, there exist several methods in the literature. In general, these methods can be divided into two groups. Some of them consider the image to be reduced in a global way [1], [2] or in a transform domain [3]. Other widely used methods act locally over pieces (blocks) of the image [4], [5]. The division of the image in blocks of small size allows one to design simple reduction algorithms since the computation required is usually very low.

Grayscale image reduction methods that act over blocks are very related to aggregation functions [6], [7], since in these methods it is necessary to aggregate a set of inputs (generally the intensities of the pixels in the block) into a representative value. Aggregation functions are also very used in other topics of image processing [8]. Moreover, it is known that by minimizing penalty functions we obtain averaging aggregation functions, that are the kind of functions usually demanded in image processing.

However, when we work with color images (RGB, CMY, etc) it is not clear the way to aggregate a set of pixels. In this work we understand the RGB scheme (or other color schemes) to be a cartesian product of lattices. In this way, we study the cartesian product of lattices and the cartesian product of chains [9]-[11]. From this study, we define penalty functions defined on a product lattice. Finally, by minimizing penalty functions we obtain a family of averaging functions over a product lattice. We show that in general this averaging functions are different from applying the same averaging function componentwise.

We present an RGB color image reduction which is based on minimizing a penalty function by approximating the value using a set of possible averaging functions componentwise. We carry out an experimental study comparing different penalty functions and aggregation functions and analyzing the stability of the algorithms to different noises in the images.

The structure of the paper is as follows: first, we recall some preliminary definitions. In Section 3 we study the cartesian product of lattices and the definition of penalty functions and aggregation functions in product lattices. Then, in Section 4, we introduce our reduction algorithm for color images and we perform an experimental study of this proposal in Section 5. We finish with some conclusions and final remarks.

\section{PRELIMINARIES}

\section{A. Aggregation functions}

Definition 1: [12]-[16] A function $f:[a, b]^{n} \rightarrow[a, b]$ is called an aggregation function if it is monotone non-decreasing in each variable and satisfies $f(\mathbf{a})=a, f(\mathbf{b})=b$, with $\mathbf{a}=$ $(a, a, \ldots, a), \mathbf{b}=(b, b, \ldots, b)$.

Definition 2: An aggregation function $f$ is called averaging if it is bounded by the minimum and maximum of its arguments

$$
\begin{aligned}
& \min (\mathbf{x}):=\min \left(x_{1}, \ldots, x_{n}\right) \leq f\left(x_{1}, \ldots, x_{n}\right) \leq \\
& \leq \max \left(x_{1}, \ldots, x_{n}\right)=: \max (\mathbf{x}) .
\end{aligned}
$$

It is immediate that averaging aggregation functions are idempotent (i.e., $\forall t \in[a, b]: f(t, t, \ldots, t)=t$ ) and (because of monotonicity) vice versa. Then clearly the boundary conditions $f(\mathbf{a})=a, f(\mathbf{b})=b$ are satisfied. 
Well known examples of averaging functions are the arithmetic mean and the median. It is known that the arithmetic mean and the median are solutions to simple optimization problems, in which a measure of disagreement between the inputs is minimized, see [13], [17]-[20]. The main motivation is the following. Let $\mathbf{x}$ be the inputs and $y$ be the output. If all the inputs coincide $x=x_{1}=\ldots=x_{n}$, then the output is $y=x$, and we have a unanimous vote. If some input $x_{i} \neq y$, then we impose a "penalty" for this disagreement. The larger the disagreement, and the more inputs disagree with the output, the larger (in general) is the penalty. We look for an aggregated value which minimizes the penalty.

Thus we need to define a suitable measure of disagreement, or dissimilarity.

Definition 3: Let $P:[a, b]^{n+1} \rightarrow \Re$ be a penalty function with the properties

i) $P(\mathbf{x}, y) \geq 0$ for all $\mathbf{x}, y$;

ii) $P(\mathbf{x}, y)=0$ if all $x_{i}=y$;

iii) $P(\mathbf{x}, y)$ is quasiconvex in $y$ for any $\mathbf{x}$.

The penalty based function is

$$
f(\mathbf{x})=\arg \min _{y} P(\mathbf{x}, y),
$$

if $y$ is the unique minimizer, and $y=\frac{a+b}{2}$ if the set of minimizers is the interval $[a, b]$.

Theorem 1: [18] Any averaging aggregation function can be represented as a penalty based function in the sense of Definition 3

Example 1: The arithmetic mean is represented via the penalty function

$$
\arg \min _{y} \sum_{i=1}^{n}\left(x_{i}-y\right)^{2}
$$

and the median is represented via the penalty function

$$
\arg \min _{y} \sum_{i=1}^{n}\left|x_{i}-y\right| \text {. }
$$

\section{B. Lattices}

Definition 4: Let $L$ be a set. A lattice $\mathcal{L}=(L, \leq, \wedge, \vee)$ is a poset with the partial order $\leq$ on $L$, and meet and join operations $\wedge, \vee$, if every pair of elements from $L$ has both meet and join.

Definition 5: Let $P$ be a poset. A chain in $P$ is a totally ordered subset of $P$. The length of a chain is its cardinality.

Theorem 2: Let $\mathcal{L}_{1}=\left(L_{1}, \leq_{1}, \wedge_{1}, \vee_{1}\right)$ and $\mathcal{L}_{2}=\left(L_{2}, \leq_{2}\right.$ $\left., \wedge_{2}, \vee_{2}\right)$ be two lattices. The Cartesian product $\mathcal{L}_{1} \times \mathcal{L}_{2}=$ $\left(L_{1} \times L_{2}, \leq, \wedge, \vee\right)$ with $\leq$ defined by

$$
\left(x_{1}, y_{1}\right) \leq\left(x_{2}, y_{2}\right) \Leftrightarrow x_{1} \leq_{1} x_{2} \text { and } y_{1} \leq_{2} y_{2},
$$

and

$$
\begin{aligned}
& \left(x_{1}, y_{1}\right) \wedge\left(x_{2}, y_{2}\right)=\left(x_{1} \wedge_{1} x_{2}, y_{1} \wedge_{2} y_{2}\right) \\
& \left(x_{1}, y_{1}\right) \vee\left(x_{2}, y_{2}\right)=\left(x_{1} \vee_{1} x_{2}, y_{1} \vee_{2} y_{2}\right)
\end{aligned}
$$

is a lattice.
Notice that all finite chains of the same length are isomorph to each other, and we can represent them as non-negative integers $0,1, \ldots, K$, and elements of product lattices as tuples $x=\left(x_{1}, x_{2} \ldots, x_{m}\right), x_{i} \in Z_{+}=\{0,1,2, \ldots\}$.

\section{Penalty functions Defined on PROdUCt lattices}

In this section we first define the length between two elements in a product of finite chains and we apply this distance in the definition of penalty functions in cartesian product of lattices.

Definition 6: Let $\mathcal{L}=(L, \leq, \wedge, \vee)$ be a product of finite chains. The distance between $x, y \in \mathcal{L}$ is defined as the length of a maximal chain $\mathcal{C}$ with the least element $a=x \wedge y$ and the greatest element $b=x \vee y$ minus 1 ,

$$
d(x, y)=\operatorname{length}(\mathcal{C})-1 .
$$

Remark 1: We note that all maximal chains with the least element $a$ and the greatest element $b$ on a product lattice in Definition 6 have all the same length. This definition is equivalent to the following

$$
d(x, y)=\sum_{i=1}^{m} d_{i}\left(x_{i}, y_{i}\right)=\sum_{i=1}^{m}\left|x_{i}-y_{i}\right|,
$$

where $d_{i}$ is the distance in the $i$-th chain in the product of $m$ chains.

Definition 7: Let $\mathcal{L}$ be a product of finite chains, $x_{1}, \ldots, x_{n} \in \mathcal{L}$ and the penalty function $P: Z_{+}^{n} \rightarrow \Re$. The penalty based function on $\mathcal{L}$ is given by

$$
\begin{aligned}
& f\left(x_{1}, \ldots, x_{n}\right)=\mu= \\
& =\arg \min _{y \in \mathcal{L}} P\left(d\left(x_{1}, y\right), d\left(x_{2}, y\right), \ldots, d\left(x_{n}, y\right)\right) .
\end{aligned}
$$

The minimum always exists and $\mu \in \mathcal{L}$. There can be several minimizers. In this case one can take any minimizer.

Theorem 3: The function $f$ in Definition 7, is an averaging (and hence idempotent) function.

Proof: Clearly $\bigwedge x_{i}=a \leq \mu \leq b=\bigvee x_{i}$, because for any $x_{i}, d\left(x_{i}, a\right)<d\left(x_{i}, t\right)$ with $t<a$, and similarly at the other end.

A special case of penalty based functions was considered in [21], called dissimilarity functions (see also [22], [23]), where the penalty $P$ is given by

$$
P(\mathbf{x}, y)=\sum_{i=1}^{n} K\left(x_{i}-y\right),
$$

where $K$ is a convex function with the unique minimum $K(0)=0$. In this case the penalty based function is monotone, i.e., an aggregation function. By adapting this definition to our case we have the following result:

Theorem 4: Let the penalty function $P: Z_{+}^{n} \rightarrow \Re$ be given by

$$
P(\mathbf{x}, y)=\sum_{i=1}^{n} K\left(d\left(x_{i}, y\right)\right) .
$$

The penalty based function $f$ in the sense of Definition 7 is an averaging aggregation function on a product lattice. 
Example 2: Let $K(x)=x^{2}$ and $P(\mathbf{x}, y)=\sum_{i=1}^{n} d\left(x_{i}, y\right)^{2}$. The minimization of $P$ corresponds to the arithmetic mean and is given by

$$
\text { minimize }_{y \in \mathcal{L}} \sum_{i=1}^{n}\left(\sum_{j=1}^{m}\left|x_{i j}-y_{j}\right|\right)^{2} \text {. }
$$

where $x_{i j}$ denotes the $j$-th component of the $i$-th tuple $x_{i} \in \mathcal{L}$. Observe that the solution to this problem can be different from applying the arithmetic mean to each component $j$ (that is, the cartesian product of arithmetic means).

Example 3: Let $K(x)=|x|$ and $P(\mathbf{x}, y)=\sum_{i=1}^{n} d\left(x_{i}, y\right)$. The minimization of $P$ corresponds to the median and is given by

$$
\operatorname{minimize}_{y} \sum_{i=1}^{n} \sum_{j=1}^{m}\left|x_{i j}-y_{j}\right|=\sum_{j=1}^{m} \sum_{i=1}^{n}\left|x_{i j}-y_{j}\right| .
$$

where $x_{i j}$ denotes the $j$-th component of the $i$-th tuple $x_{i} \in \mathcal{L}$. Observe that the minimum is obtained when we take $y=$ $\left(\operatorname{Med}\left(x_{\cdot, 1}\right), \ldots, \operatorname{Med}\left(x_{\cdot, m}\right)\right)$, that is, the cartesian product of the medians.

\section{IMAGE REDUCTION ALGORITHM BY MINIMIZING PENALTY FUNCTIONS}

In image processing, we understand colors as a product of finite chains. When we deal with the RGB color scheme specifically, the length of each finite chain is typically 256. Having this in mind, we can use the concept of penalty function in cartesian product of lattices defined in Section III when we need to aggregate several pixels of a color image.

In this section we present a color image reduction algorithm based on the minimization of penalty functions. The algorithm is based on dividing the image into disjoint blocks. Then, we apply an averaging aggregation function to the pixels in each block by minimizing the penalty function. Remind that, in general, the aggregation function in product lattices are not built as the cartesian product of the corresponding aggregation function in each channel. For this reason, we fix a number of $k$ different averaging aggregation function. We apply the aggregation functions to each of the blocks of the image (componentwise) obtaining a set of $k$ possible solutions. The pixel in the reduced image corresponds to the solution with minimum penalty with respect to the inputs. In Algorithm 1 and Figure 1 we show the scheme of the algorithm and a diagram illustrating it.

Example 4: Consider the following block of an image in RGB:

\begin{tabular}{|lll}
\multicolumn{1}{c}{$\mathbf{x}_{R}$} \\
\hline 10 & 15 & 15 \\
12 & 12 & 13 \\
10 & 11 & 11 \\
\hline & $\mathbf{x}_{B}$ \\
\hline 221 & 220 & 220 \\
150 & 200 & 210 \\
215 & 200 & 215 \\
\hline
\end{tabular}

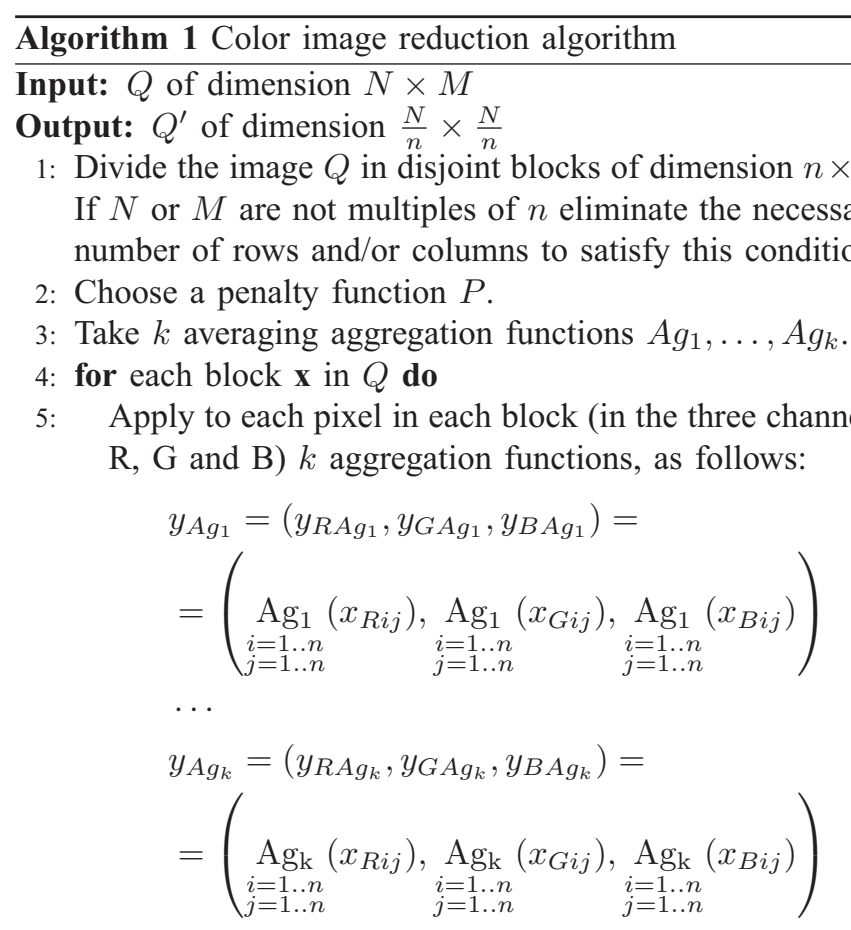

6: $\quad$ Calculate the penalties $P_{i}=P\left(\mathbf{x}, y_{A g_{i}}\right)$ for each $\mathbf{y}_{A g_{i}}$ with $i=1, \ldots, k$.

7: Assign the value $\mathbf{y}_{A g_{i}}$ with the smallest penalty to the corresponding pixel of the reduced image.

$$
\arg \min _{y_{A g_{i}}} P\left(\mathbf{x}, y_{A g_{i}}\right)
$$

8: end for

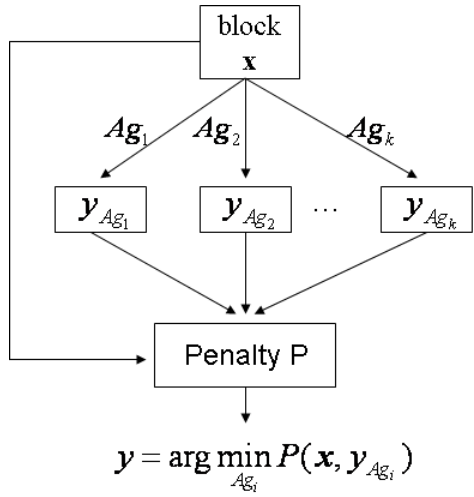

Fig. 1. Scheme of Algorithm 1

Suppose we take the penalty function corresponding to the arithmetic mean given by:

$$
P(\mathbf{x}, y)=\sum_{i=1}^{3} \sum_{j=1}^{3}\left(\sum_{C \in\{R, G, B\}}\left|x_{C i j}-y_{C}\right|\right)^{2}
$$

We take a set of $k$ averaging aggregation functions given by: minimum ( $\mathrm{min})$, geometric mean (geom), arithmetic mean (arith), median (med) and maximum (max). We apply these functions to the block (componentwise) obtaining a set of $k$ 


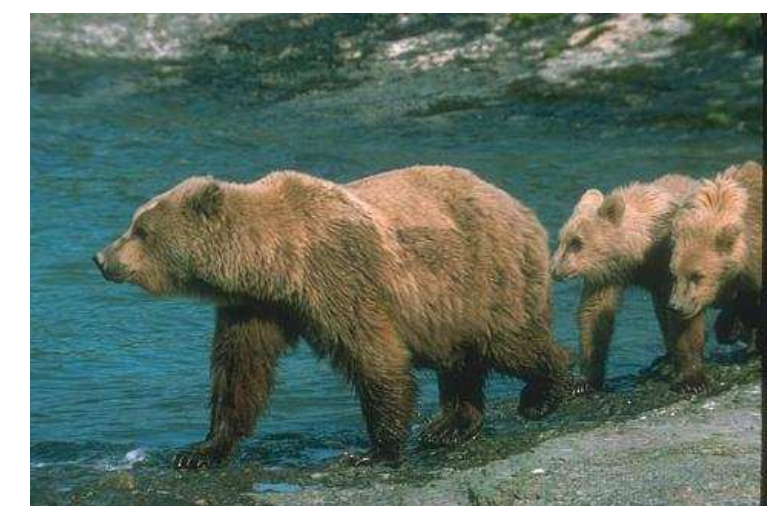

$a$

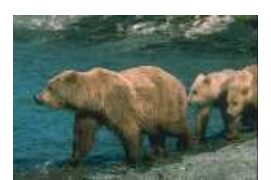

$b$

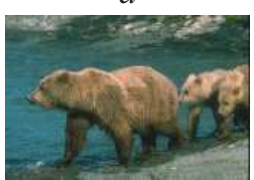

$c$

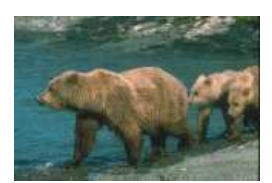

$d$
Fig. 2. Original image (image $a$ ) and 3 reductions obtained by applying Algorithm 1 with $K(x)=|x|$ (image $b$ ), $K(x)=x^{2}$ (image $c$ ) and $K(x)=$ $x^{2}$ (image $d$ ).

possible solutions.

$$
\begin{aligned}
y_{\text {min }} & =(10,100,150) \\
y_{\text {geom }} & =(11.98,122.9,204.41) \\
y_{\text {arith }} & =(12.11,123.33,205.67) \\
y_{\text {med }} & =(12,125,215) \\
y_{\max } & =(15,135,220)
\end{aligned}
$$

Applying the penalty function to the set of solutions, we get:

$$
\begin{aligned}
P\left(\mathbf{x}, y_{\text {min }}\right) & =62520 \\
P\left(\mathbf{x}, y_{\text {geom }}\right) & =7904 \\
P\left(\mathbf{x}, y_{\text {arith }}\right) & =7714 \\
P\left(\mathbf{x}, y_{\text {med }}\right) & =7364 \\
P\left(\mathbf{x}, y_{\text {max }}\right) & =10920
\end{aligned}
$$

So the pixel that represents the whole block is given by the minimum penalty $y_{\text {med }}=(12,125,215)$.

In Figure 2 we illustrate the behavior of Algorithm 1 with an original RGB color image of dimension $321 \times 481$ pixels. We take $n=3$, so the reduced images after applying Algorithm 1 have $107 \times 160$ pixels (observe that 481 is not multiple of $n=3$, so we have deleted one column of the original image). The aggregation functions used are the same as in Example 4 and as penalty functions we take $K(x)=|x|$ (image b of Fig. 2), $K(x)=x^{2}$ (image c of Fig. 2) and $K(x)=\left|x^{3}\right|$ (image d of Fig. 2).

\section{A. Frequency of averaging aggregation functions}

We are interested in analyzing the frequency of each aggregation function in Algorithm 1 with some penalty functions.
TABLE I

FREQUENCY OF CHOOSING EACH AGGREGATION FUNCTION IN IMAGE $a$ OF FIG. 2

\begin{tabular}{|l||c||c||c||c||c|}
\hline & min & geom & arith & med & max \\
\hline$K(x)=|x|$ & $0.00 \%$ & $0.00 \%$ & $0.00 \%$ & $100.00 \%$ & $0.00 \%$ \\
\hline$K(x)=x^{2}$ & $0.05 \%$ & $5.55 \%$ & $80.22 \%$ & $14.17 \%$ & $0.00 \%$ \\
\hline$K(x)=\left|x^{3}\right|$ & $0.05 \%$ & $36.23 \%$ & $54.81 \%$ & $8.88 \%$ & $0.00 \%$ \\
\hline
\end{tabular}

TABLE II

FREQUENCY OF CHOOSING EACH AGGREGATION FUNCTION IN THE REDUCED IMAGES OF IMAGE $a$ IF FIG. 3

\begin{tabular}{|l||c||c||c||c||c|}
\hline & min & geom & arith & med & max \\
\hline$K(x)=|x|$ & $0.00 \%$ & $0.00 \%$ & $0.00 \%$ & $100.00 \%$ & $0.00 \%$ \\
\hline$K(x)=x^{2}$ & $0.00 \%$ & $9.23 \%$ & $40.77 \%$ & $50.00 \%$ & $0.00 \%$ \\
\hline$K(x)=\left|x^{3}\right|$ & $0.12 \%$ & $6.40 \%$ & $66.85 \%$ & $26.50 \%$ & $0.13 \%$ \\
\hline
\end{tabular}

Recall that if we take $K(x)=|x|$ in Theorem 4, then we always take the median as the aggregation function. We analyze the percentage of each aggregation function when we take $K(x)=x^{2}$ and $K(x)=\left|x^{3}\right|$. For this purpose, we repeat the same experiment as in Example 4 with an RGB color image of dimension obtained from [24] (see Fig. 2)

Remark 1: Observe that if all the pixels in one block have the same intensity, then any averaging aggregation function minimizes the penalty function. However, in the image of Fig. 2 there are any block satisfying this condition.

Next, we analyze the reaction of Algorithm 1 to the presence of impulsive noise (salt and pepper) in the original image. For this reason, we modify image $a$ of Fig. 2 altering $10 \%$ of pixels. In Fig. 3 we show the altered image with noise (image $a$ ) and three reduced images taking $K(x)=|x|$ (image $b$ ), $K(x)=x^{2}$ (image $c$ ) and $K(x)=\left|x^{3}\right|$ (image $d$ ). We visually observe that if we take $K(x)=|x|$, the reduced image obtained has less quantity of noise because we always take the median (and we know that the median allows to filter extreme values of impulsive noise). Moreover, observe that image $c$ has better quality (less noise) than image $d$ because when we take $K(x)=x^{2}$, the frequency of choosing the median (in comparison with the arithmetic mean) increases highly (and increases more than with $K(x)=\left|x^{3}\right|$ ). The percentage of choice of each operator can be seen in Table II and graphically in in Fig. 4 for the median and the arithmetic mean.

\section{EXPERIMENTAL RESULTS}

In this section we propose an experimental study of the performance of Algorithm 1 with 3 different penalty functions: taking $K(x)=|x|(\operatorname{alg} 1)$, taking $K(x)=x^{2}$ (alg2) and taking $K(x)=\left|x^{3}\right|$ (alg3); and 2 other reduction algorithms: classical subsampling (sub) that consists in taking only one pixel randomly from the block (when the size of the block is odd, we always take the central pixel) and taking the cartesian product of arithmetic mean (arith) that consists in applying the arithmetic mean componentwise to the channels R, G and B. Again, we have taken 5 images of [24] for the study (see Fig. 5).

The methodology of the study is the following: we take an image of dimension $321 \times 480$ pixels and we reduce it to 


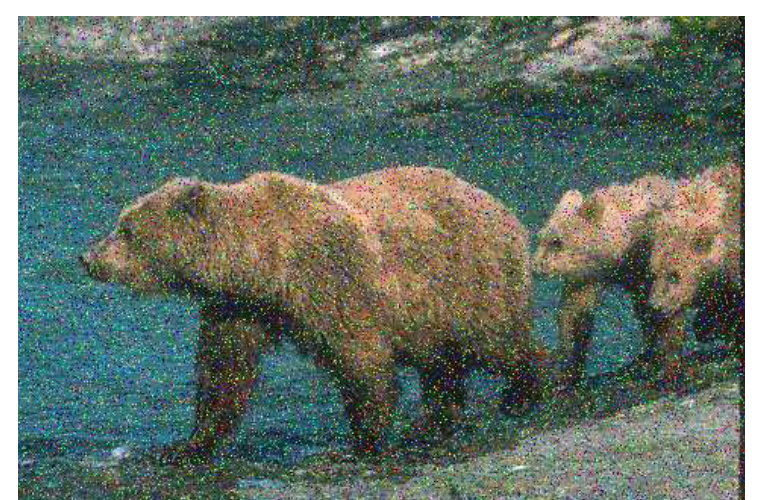

$a$

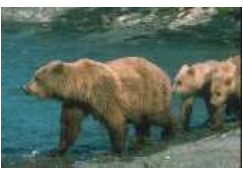

$b$

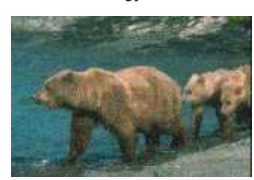

$c$

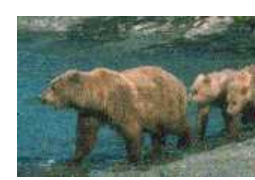

$d$
Fig. 3. Original image with $10 \%$ of impulsive noise(image $a$ ) and 3 reductions obtained by applying Algorithm 1 with $K(x)=|x|$ (image $b$ ), $K(x)=x^{2}$ (image $c$ ) and $K(x)=\left|x^{3}\right|$ (image $d$ ).
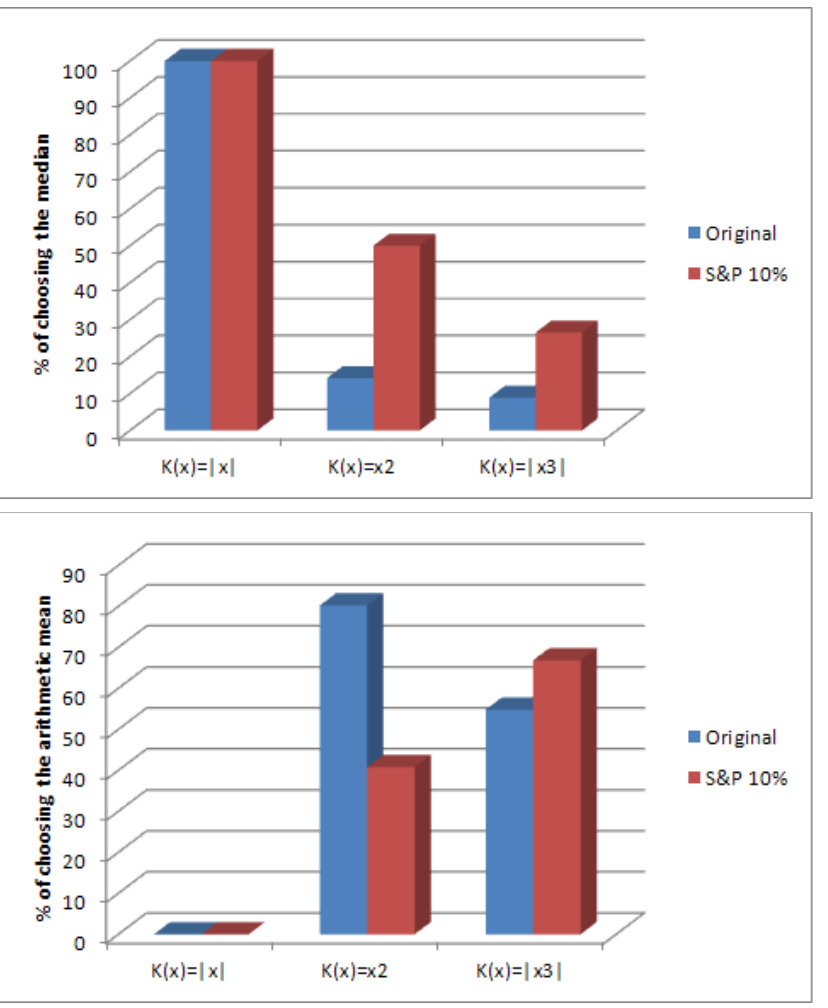

Fig. 4. Variation in the frequency of the median (up) and the arithmetic mean (down) in Algorithm1 under presence of impulsive noise
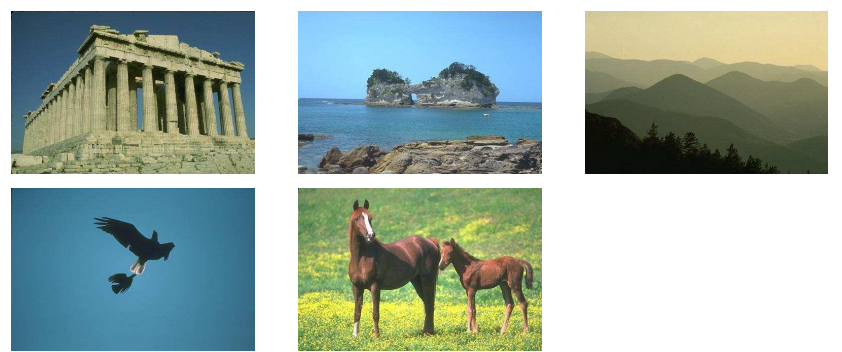

Fig. 5. Set of original images used in the experimental study

TABLE III

PSNR OF THE 5 ORIGINAL IMAGES AND RECONSTRUCTION

\begin{tabular}{|c||c||c||c||c||c|}
\hline & alg1 & alg2 & alg3 & sub & arith \\
\hline $\operatorname{Im} 1$ & 23.6935 & 24.2310 & 24.2053 & 22.3517 & 24.2337 \\
\hline $\operatorname{Im} 2$ & 25.3412 & 25.7811 & 25.7611 & 23.8045 & 25.7852 \\
\hline $\operatorname{Im} 3$ & 36.6529 & 37.1572 & 37.1422 & 35.4744 & 37.1628 \\
\hline $\operatorname{Im} 4$ & 34.2398 & 34.8538 & 34.8098 & 33.2333 & 34.8605 \\
\hline $\operatorname{Im} 5$ & 22.6243 & 23.0059 & 22.9890 & 20.9515 & 23.0137 \\
\hline
\end{tabular}

$107 \times 160$ pixels (taking $n=3$ ) by the five algorithms studied: alg1, alg2, alg3, sub and arith. Then we reconstruct (enlarge) the image to its original size $(321 \times 480)$ and we compare the similarity between the original and the reconstruction. To enlarge the image we propose to use the simplest method: to copy the value of the pixel into all the pixels of the new block. We have chosen this method because its computational cost is very poor and it allows to compare the images visually without introducing any distortion in the reconstructed image. However, some other methods can be used to increase the quality of the resulted image, as for instance [25]. Finally, we measure the similarity between two images taking the PSNR, since it is the most used and popular measure in the literature. The formula of the PSNR is given by: let $A, B$ be two RGB images of dimension $N \times M$

$$
\begin{aligned}
\operatorname{PSNR}(A, B) & =10 \cdot \log _{10}\left(\frac{255^{2}}{M S E(A, B)}\right) \text { with } \\
\operatorname{MSE}(A, B) & =\frac{\sum_{i=1}^{N} \sum_{j=1}^{M} \sum_{c \in\{R, G, B\}}\left(A_{c i j}-B_{c i j}\right)^{2}}{N \cdot M \cdot 3}
\end{aligned}
$$

In Table III we show the PSNR of the 5 original images and the reconstructed images after reducing it with the 5 studied methods. Observe that the best results are achieved by arith because the mean squared error (and therefore, the PSNR) is maximized when the arithmetic mean is taken as the reduction operator (as a consequence of Example 1). These results are very similar to the obtained by alg2 because, as it is mentioned in Section IV-A, the arithmetic mean is aggregation operator most frequently chosen. Observe also that sub obtains the worst results for all the images.

We now consider the same images altered with salt and pepper noise. We have changed $10 \%$ of the pixels in test images. We repeat the previous experiment over this set of images. In Table IV we show the PSNR obtained between the reconstucted images and the original ones without noise. With these results, we see that the best results are obtained 
alg1

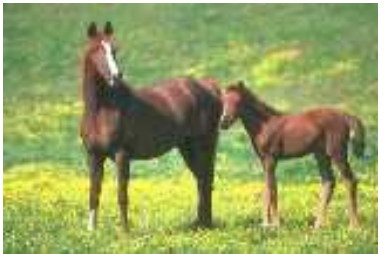

alg3

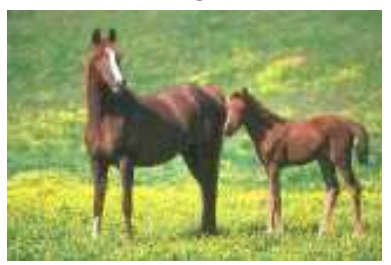

arith

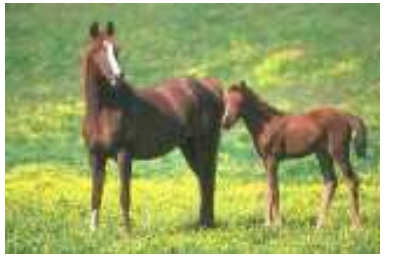

$\operatorname{alg} 2$

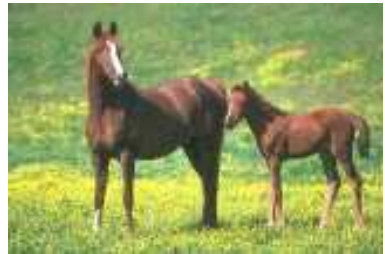

sub

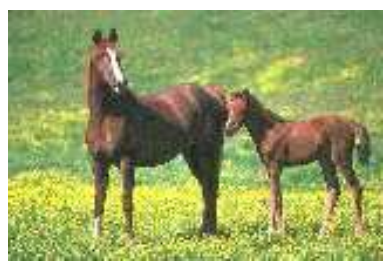

Fig. 8. Reduced images obtained after applying the 5 studied methods to an image altered with gaussian noise.

TABLE IV

PSNR OF THE 5 ORIGINAL IMAGES WITH AND RECONSTRUCTION AFTER ADDING $10 \%$ OF SALT AND PEPPER NOISE TO ORIGINAL IMAGES

\begin{tabular}{|c||c||c|c||c||c|}
\hline & alg1 & alg2 & alg3 & sub & arith \\
\hline Im1 & 23.4343 & 22.6209 & 21.9416 & 14.6701 & 21.3733 \\
\hline Im2 & 25.0471 & 23.5167 & 22.3168 & 14.5178 & 21.7449 \\
\hline Im3 & 36.1647 & 27.1392 & 24.9765 & 14.8780 & 23.4846 \\
\hline Im4 & 33.9061 & 28.3872 & 25.9676 & 15.5453 & 24.3319 \\
\hline Im5 & 22.3758 & 21.6776 & 21.1308 & 14.3721 & 20.6572 \\
\hline
\end{tabular}

by alg1. This behaviour is due to with alg1 we always take the median and this operator is known to be suitable for this impulsive noise. Moreover, the results of alg2 are also very good because as we can see in Section IV-A, the frequency of choosing the median (instead of the arithmetic mean) is increased when impulsive noise is added to the images. This fact is also observable in alg3, but the median is still less frequent. Notice that our proposals outperform the results of sub and arith for all the images.

Finally, we alter the same images with additive gaussian noise $(\sigma=10)$. We a priori know that the arithmetic mean is the most suitable operator for this kind of noise. In Table $\mathrm{V}$ we show the result of this experiment where arith achieves the best results as it was expected. However, alg2 and alg3 get PSNR values almost as good as arith. This indicates that these penalty functions are also suitable for this kind of noise.

Although there are reduction methods that get the best solution for a specific kind of noise presented in the images, from these results we have proved that our reduction algorithm taking $K(x)=x^{2}(\operatorname{alg} 2)$ and $K(x)=x^{3}$ (alg3) is very robust when two different kind of noises are present.

Fig. 7. Reduced images obtained after applying the 5 studied methods to an image altered with impulsive noise. 
TABLE V

PSNR OF THE 5 ORIGINAL IMAGES WITH AND RECONSTRUCTION AFTER ADDING ADDITIVE GAUSSIAN NOISE TO ORIGINAL IMAGES WITH $\sigma=10$

\begin{tabular}{|c||c||c||c||c||c|}
\hline & alg1 & alg2 & alg3 & sub & arith \\
\hline $\operatorname{Im} 1$ & 23.5128 & 23.9547 & 23.9698 & 21.3478 & 24.0410 \\
\hline $\operatorname{Im} 2$ & 25.0471 & 25.4148 & 25.4302 & 22.5206 & 25.4939 \\
\hline $\operatorname{Im} 3$ & 33.3510 & 33.8370 & 34.0965 & 27.5104 & 34.4620 \\
\hline $\operatorname{Im} 4$ & 32.0463 & 32.6079 & 32.7568 & 26.9464 & 33.0377 \\
\hline $\operatorname{Im} 5$ & 22.4417 & 22.7943 & 22.8080 & 20.2316 & 22.8674 \\
\hline
\end{tabular}

In Figures 6,7 and 8 we show the reduced version of 3 test images obtained by the 5 reduction algorithms studied. Figure 6 corresponds to applying the algorithms to an unaltered original image. Figure 7 is obtained after altering an image with salt and pepper noise (10\%) while Figure 8 is obtained after alteryng an image with gaussian noise $(\sigma=10)$.

\section{CONCLUSIONS}

In this work we have defined penalty functions and, by minimizing them, aggregation functions on product lattices. With these constructions, we have defined a way of aggregating color values in image processing. This has led us to propose an image reduction algorithm of RGB color images by minimizing penalty functions. We have studied that the penalty functions proposed obtain very good results and are very robust to the presence of different type of noises present in images.

\section{ACKNOWLEDGMENT}

This work has been partially supported by grants TIN201015055 and TIN2011-29520 of the Government of Spain.

\section{REFERENCES}

[1] I. Perfilieva, "Fuzzy transforms: Theory and applications," Fuzzy Sets and Systems, vol. 157, pp. 993-1023, 2006.

[2] H. Nobuhara, K. Hirota, S. Sessa, and W. Pedrycz, "Efficient decomposition methods of fuzzy relation and their application to image decomposition," Applied Soft Computing, vol. 5, pp. 399-408, 2005.

[3] Y. Park and H. Park, "Arbitrary-ratio image resizing using fast dct of composite length for dct based transcode," IEEE Transactions on Image Processing, vol. 15, pp. 494-500, 2006.

[4] F. Di Martino, V. Loia, I. Perfilieva, and S. Sessa, "An image coding/decoding method based on direct and inverse fuzzy transforms," International Journal of Approximate Reasoning, vol. 48, pp. 110-131, 2008.

[5] V. Loia and S. Sessa, "Fuzzy relation equations for coding/decoding processes of images and videos," Information Sciences, vol. 171, pp. 145-172, 2005

[6] D. Paternain, C. Lopez-Molina, H. Bustince, R. Mesiar, and G. Beliakov, "Image reduction using fuzzy quantifiers," Advances in Intelligen and Soft Computing, vol. 107, pp. 351-362, 2011.

[7] G. Beliakov, H. Bustince, and D. Paternain, "Image reduction using means on discrete product lattices," IEEE Transactions on Image Processing, vol. 21, pp. 1070-1083, 2012.

[8] M. Galar, J. Fernandez, G. Beliakov, and H. Bustince, "Interval-valued fuzzy sets applied to stereo matching of color images," IEEE Transactions on Image Processing, vol. 20, pp. 1949-1961, 2011.

[9] B. De Baets and R. Mesiar, "Triangular norms on product lattices," Fuzzy Sets and Systems, vol. 104, pp. 61-75, 1999.

[10] S. Jenei and B. De Baets, "On the direct decomposability of t-norms on product lattices," Fuzzy Sets and Systems, vol. 139, pp. 699-707, 2003.

[11] G. Mayor and J. Monreal, "Additive generators of discrete conjunctive aggregation operations," IEEE Trans. on Fuzzy Systems, vol. 15, pp. 1046-1052, 2007
[12] G. Beliakov, A. Pradera, and T. Calvo, Aggregation Functions: A Guide for Practitioners. Heidelberg, Berlin, New York: Springer, 2007.

[13] Y. Torra, V. Narukawa, Modeling Decisions. Information Fusion and Aggregation Operators. Berlin, Heidelberg: Springer, 2007.

[14] T. Calvo, G. Mayor, and R. Mesiar, Eds., Aggregation Operators. New Trends and Applications. Heidelberg, New York: Physica-Verlag, 2002.

[15] M. Grabisch, J.-L. Marichal, R. Mesiar, and E. Pap, Aggregation Functions. Cambridge: Cambridge University press, 2009.

[16] H. Bustince, T. Calvo, B. De Baets, J. Fodor, R. Mesiar, J. Montero, D. Paternain, and A. Pradera, "A class of aggregations functions encompassing two-dimensional owa operators," Information Sciences, vol. 180, pp. 1977-1989, 2010.

[17] C. Gini, Le Medie. Milan (Russian translation, Srednie Velichiny, Statistica, Moscow, 1970): Unione Tipografico-Editorial Torinese, 1958.

[18] T. Calvo and G. Beliakov, "Aggregation functions based on penalties," Fuzzy Sets and Systems, vol. 161, pp. 1420-1436, 2010.

[19] T. Calvo, R. Mesiar, and R. Yager, "Quantitative weights and aggregation," IEEE Trans. on Fuzzy Systems, vol. 12, pp. 62-69, 2004.

[20] R. Yager and A. Rybalov, "Understanding the median as a fusion operator,” Int. J. General Syst., vol. 26, pp. 239-263, 1997.

[21] R. Mesiar, "Fuzzy set approach to the utility, preference relations, and aggregation operators," Europ. J. Oper. Res., vol. 176, pp. 414-422, 2007.

[22] H. Bustince, E. Barrenechea, and M. Pagola, "Restricted equivalence functions," Fuzzy Sets and Systems, vol. 157, pp. 2333-2346, 2006.

[23] H. Bustinces, E. Barrenechea, and M. Pagola, "Relationship between restricted dissimilarity functions, restricted equivalence functions and normal e-n-functions: Image thresholding invariant," Pattern Recognition Letters, vol. 29, pp. 525-536, 2008.

[24] D. Martin, C. Fowlkes, D. Tal, and J. Malik, "A database of human segmented natural images and its application to evaluating segmentation algorithms and measuring ecological statistics," in Proc. 8th Int'l Conf. Computer Vision, vol. 2, July 2001, pp. 416-423.

[25] A. Jurio, M. Pagola, R. Mesiar, G. Beliakov, and H. Bustince, "Image magnification using interval information," IEEE Transactions on Image Processing, vol. 20, pp. 3112-3123, 2011. 